\title{
High frequency of BRAF mutations in mucinous ovarian carcinoma of Taiwanese patients
}

\author{
Wan-Ru MD, PhD Chao \\ Chung-Shan Medical University Hospital \\ Yi-Ju Li, Li \\ Chung Shan Medical University and Hospital \\ Ming-Yung PhD Lee \\ Providence University \\ Gwo-Tarng PhD Sheu \\ Chung-Shan Medical University \\ Chih-Ping Han ( $\sim$ hanhaly2@gmail.com ) \\ Chung Shan Medical University and Hopsital
}

\section{Research article}

Keywords: mucinous ovarian carcinoma, BRAF gene, mutation

Posted Date: September 17th, 2020

DOl: https://doi.org/10.21203/rs.3.rs-70712/v1

License: (c) (i) This work is licensed under a Creative Commons Attribution 4.0 International License. Read Full License 


\section{Abstract \\ Background}

In view of the encouraging clinical evidence of $B R A F$ inhibitors that can treat some melanoma patients successfully, we aimed to investigate the status of $B R A F$ mutations of primary mucinous ovarian carcinomas (MOC) in Taiwanese women, and apply the emerging paradigm classification of BRAF mutation groups.

\section{Methods}

DNA was extracted from micro-dissected tissue samples using the QIAamp® DNA FFPE Kit. The mutations of activation segment (exon 15), CR3 (conserved regions 3), kinase domain of the BRAF gene were analyzed using the highly sensitive $B R A F$ mutant enriched kit (FemtoPath ${ }^{\circledR}$ ) with subsequent Sanger sequencing method. Additionally, we extended our prior data of HER2 aberrations and KRAS mutation into this study in order to compare with the status of BRAF mutation.

\section{Results}

Out of the 20 cases tested, $16(80 \%)$ harbored $B R A F$ missense mutations. Their mutation profile and case number ( $n$ ) were categorized as (1) class I: V600E $(n=1)$, V600M $(n=1)$; $(2)$ class II: A598V $(n=1)$, T599| $(\mathrm{n}=10)$; (3) class III: none $(\mathrm{n}=0)$; and (4) unclassified variants: S602F $(\mathrm{n}=2)$, dual T599I plus S602F $(\mathrm{n}=$ 1). The $B R A F$ missense mutation ( $(\mathrm{S} 602 \mathrm{~F}$ ) is novel. In addition, the prevalence of $B R A F$ gene mutation is significantly higher than $H E R 2$ gene mutation ( $80 \%$ vs. $35 \% ; \mathrm{p}=0.022$,) and $H E R 2$ gene amplification ( $80 \%$ vs. $35 \% ; p=0.022)$, respectively. However, the mutation rates of $B R A F$ and $K R A S$ were not significantly different ( $80 \%$ vs. $60 \% ; p=0.289)$.

\section{Discussion}

Our results showed that $B R A F$ mutation is not uncommon in primary MOC of Taiwanese. When taken together with previous published data, we found that the activating $B R A F$ mutation, $H E R 2$ amplification, HER2 mutation and KRAS mutation were not mutually exclusive, but simultaneously independent. However, they may even have a synergistic effect in tumorigenesis.

\section{Conclusions}

The BRAF variant with T599I stands the majority. These findings suggested that there was a lower potential response to the existing $V 600$ BRAF inhibitors, but may be responsive to dual BRAF plus MEK inhibitors or single MEK inhibitor in MOC. Further studies are warranted to investigate the clinical benefits 
of newly targeted therapy in recurrent or advanced stage MOC patients carrying each class of $B R A F$ mutation.

\section{Background}

After careful exclusion of metastatic diseases, primary mucinous ovarian carcinoma (MOC) comprised less than $3 \%$ of all epithelial ovarian cancers. For years, there has been much discussion that MOC seems to be a unique disease that responds poorly to conventional chemotherapy regimens. Even though long survival and favorable outcome can occur in general with early diagnosis and optimal operation, recurrent and advanced disease are associated with poor prognosis. So far, neither modern guidelines, nor therapeutic consensus existed for the best management of recurrent or advanced MOC. [1-3]

$B R A F$, a member of the rapidly accelerated fibrosarcoma (RAF) kinase family, is able to transduce signals downstream of $R A S$ via the mitogen-activated protein kinase (MAPK) pathway. Under physiologic conditions, this pathway is tightly regulated through a negative feedback loop [4]. Activating mutations of $B R A F$ can autonomously lead to uncontrolled cellular proliferation and cell survival. The anti-BRAF drugs are successfully used in clinical practice for melanoma and achieve favorable responses for low-grade serous ovarian carcinoma $[5,6]$. Based on that information, we aimed to explore the BRAF mutation status and evaluate whether it can be a potential therapeutic target for patients with recurrent or advanced stage MOC.

Based on the mechanisms for activation of the MAPK pathway, a new model categorized the BRAF mutations into the following: (1) class I variants are V600 mutations, monomer with high level of kinase activity and $R A S$ signaling independence, (2) class II variants are non-V600 mutations, dimer with intermediate to high level of kinase activity and $R A S$ signaling independence, (3) class III variants are nonV600 mutations, dimer with absent or impaired kinase activity and $R A S$ signaling dependence, and (4) unclassified variants are other $R A F$ mutations that having unknown function. [7]

Currently, we not only investigated the mutational status of BRAFMOC in Taiwanese women, but also extended our prior data of HER2 aberrations and KRAS mutation of all samples tested $(\mathrm{n}=20)$ to this study. After that, we can (1) assess the pairwise comparison of $B R A F$ mutation with the respective KRAS mutation, HER2 mutation and HER2 amplification; (2) evaluate the prevalence of $B R A F$-based dual, triple and quadruple mutation sets including the co-existing BRAF mutation with KRAS mutations, HER2 mutations and HER2 amplifications; (3) determine whether those MOC with wild-type HER2 and KRAS can alternatively activate the MAPK signaling pathway through $B R A F$ mutation; and (4) apply the emerging paradigm classification system for $B R A F$ mutations and predict the potential implication of anti-BRAF therapeutic strategy in MOC.

\section{Methods}


Originally, we had $21 \mathrm{MOC}$ tissue specimens left over from previous studies. However, except for one case missing residual DNA and lacking of enough extra tumor component, the characteristics of all remaining study materials of all 20 cases of MOC were described in our previous report, including tissue retrieval and DNA preparation. [2, 8-10] Additionally, 7 normal ovarian tissues are used as negative controls. The research was conducted according to International Conference on Harmonization guidelines and complied with all applicable regulations for protection of human subjects of research, including review and approval by the Institutional Review Board, Chung-Shan Medical University Hospital Taichung, Taiwan.

In this study, we used the FemtoPath BRAF Mutation Screen Kit, also named Medaysis Ultra-Sensitive BRAF Mutation Detection Kit. The Medaysis Inc. US is the delegation agent of the HongJing Inc. TW in the United States. Since the Medaysis business model should use its own brand in US, Femtopath's products need to be changed to Medaysis labels in their own brand names. This Kit applies a CloDiA ${ }^{\text {Tm }}$ PCR method by means of novel and proprietary mutation enrichment technology, in which two types of skills are involved - Unindel ${ }^{T M}$ PCR and Stuntmer ${ }^{T M}$ PCR. $[11,12]$ Uninde ${ }^{T M} P C R$ is designed to identify a broad range of insertions/deletions (universal insertions/deletions) in the target region. Stuntmer ${ }^{\text {TM }}$ PCR can detect a broad range of point mutations in sequence before and after V600 hot spots (amino acid range 591-620) in exons 15 of human BRAF gene. $[13,14]$

Briefly, the sample nucleic acid with the mutation sequence is preferentially amplified over the wild type sequence by the self-competitive primers. PCR products were sequenced by the Sanger sequencing technique.

The pathogenicity associated with each $B R A F$ mutations were identified in accordance with data of the Catalogue of Somatic Mutations in Cancer (COSMIC). The functional effect of novel BRAF missense mutations can be calculated using the Web software server Polymorphism Phenotyping v2 (PolyPhen-2), which can predict the possible impact of amino acid substitutions on the stability and function of human proteins using structural and comparative evolutionary considerations. [15]

McNemar's test was used to assess the significance of the difference between 2 paired dichotomous oncogenomic status, including (1) BRAF mutation rates vs. KRAS mutation rates; (2) BRAF mutation rates vs. HER2 mutation rates; and (3) BRAF mutation rates vs. HER2 amplification rates, individually. Data were analyzed using standard statistical software, version 9.0 (SPSS, Inc., Chicago, IL). The test was 2sided and the significance level was 0.05 .

\section{Results}

In this research, we found 4 cases (20\%) with wild type BRAF and 16 cases (80\%) with BRAF somatic missense mutations in all 20 cases tested. Of the 16 BRAF mutants, 1 had double missense mutations (T599I and S602F); 1 had a single missense mutation with an additional silent mutation (T599I and V600V); as well as 14 had single missense mutation including T599I $(n=9), A 598 V(n=1), \operatorname{V600E}(n=1)$, $\operatorname{V600M~}(n=1)$ and S602F $(n=2)$. The missense mutation (S602F) is novel. All data of BRAF mutation 
analysis were based on the Catalogue of Somatic Mutations in Cancer (COSMIC) database. The locations and types of $B R A F$ mutations are presented in Table 1 and Fig. 1. Neither insertion, nor deletion of $B R A F$ gene was detected. On the other hand, no BRAF mutations were detected in the 7 normal ovarian tissues. In addition, our previously published raw data of HER2 amplifications, HER2 mutations and KRAS mutations of all samples tested are integrated in Table 1. 
Table 1

Oncogenic alteration analysis of MOC in twenty cases

\begin{tabular}{|c|c|c|c|c|}
\hline $\begin{array}{l}\text { Case } \\
\text { number }\end{array}$ & $\begin{array}{l}\text { Her2 gene } \\
\text { mutation }\end{array}$ & $\begin{array}{l}\text { Her2 gene } \\
\text { amplified }\end{array}$ & KRAS gene mutation & $\begin{array}{l}\text { BRAF gene } \\
\text { mutation }\end{array}$ \\
\hline 1 & Wild type & amplified & c. $32 \mathrm{C}>\mathrm{T}, \mathrm{p} . \mathrm{A} 11 \mathrm{~V}$ & $\begin{array}{l}\text { c. } 1796 C> \\
\text { T.p.T599| }\end{array}$ \\
\hline 2 & $\begin{array}{l}\text { c. } 2287 G> \\
\text { A.p.A763T }\end{array}$ & Non-amplified & Wild type & $\begin{array}{l}\text { c. } 1796 \mathrm{C}> \\
\text { T,p.T599I }\end{array}$ \\
\hline \multirow[t]{2}{*}{3} & \multirow[t]{2}{*}{$\begin{array}{l}\text { c. } 2938 G> \\
\text { A,p.A980T }\end{array}$} & \multirow[t]{2}{*}{ Non-amplified } & \multirow[t]{2}{*}{ Wild type } & $\begin{array}{l}\text { c.1796C>> } \\
\text { T,p.T599l }\end{array}$ \\
\hline & & & & $\begin{array}{l}\text { c. } 1800 \mathrm{G}>\mathrm{A}, \mathrm{p} . \\
\text { V600V }\end{array}$ \\
\hline 4 & $\begin{array}{l}\text { c.2329G> } \\
\text { T.p.V777L }\end{array}$ & amplified & Wild type & $\begin{array}{l}\text { c. } 1793 \mathrm{C}> \\
\text { T,p.A598V }\end{array}$ \\
\hline 5. & Wild type & Non-amplified & c.35G > A,p.G12D & $\begin{array}{l}\text { c. } 1796 \mathrm{C}> \\
\text { T,p.T599l }\end{array}$ \\
\hline 6 & Wild type & Non-amplified & c. $35 G>A, p . G 12 D$ & Wild type \\
\hline 7 & Wild type & Non-amplified & c.35G > A,p.G12D & $\begin{array}{l}\text { c. } 1796 \mathrm{C}> \\
\text { T.p.T599l }\end{array}$ \\
\hline 8 & Wild type & Non-amplified & c. $32 \mathrm{C}>\mathrm{T}, \mathrm{p} . \mathrm{A} 11 \mathrm{~V}$ & $\begin{array}{l}\text { c. } 1799 \mathrm{~T}> \\
\text { A,p.V600E }\end{array}$ \\
\hline 9 & Wild type & Non-amplified & c. $35 \mathrm{G}>\mathrm{T}, \mathrm{p} . \mathrm{G} 12 \mathrm{~V}$ & $\begin{array}{l}\text { c. } 1796 C>> \\
\text { T,p.T599| }\end{array}$ \\
\hline 10 & $\begin{array}{l}\text { c. } 2555 T> \\
\text { G,p.L852R }\end{array}$ & Non-amplified & c. $35 \mathrm{G}>$ c,p.G12A & Wild type \\
\hline 11 & $\begin{array}{l}\text { c. } 2560 A> \\
\text { G,p.K854E }\end{array}$ & Non-amplified & c. $35 \mathrm{G}>\mathrm{T}, \mathrm{p} . \mathrm{G} 12 \mathrm{~V}$ & $\begin{array}{l}\text { c. } 1805 \mathrm{C}> \\
\text { T,p.S602F }\end{array}$ \\
\hline 12 & Wild type & amplified & Wild type & $\begin{array}{l}\text { c. } 1796 \mathrm{C}> \\
\text { T.p.T599l }\end{array}$ \\
\hline 13 & Wild type & Non-amplified & c. $35 \mathrm{G}>\mathrm{T}, \mathrm{p} . \mathrm{G} 12 \mathrm{~V}$ & $\begin{array}{l}\text { c. } 1796 C>> \\
\text { T,p.T599| }\end{array}$ \\
\hline 14 & Wild type & Non-amplified & c. $35 G>A, p . G 12 D$ & $\begin{array}{l}\text { c. } 1796 \mathrm{C}> \\
\text { T.p.T599l }\end{array}$ \\
\hline \multirow[t]{2}{*}{15} & \multirow[t]{2}{*}{ Wild type } & \multirow[t]{2}{*}{ Non-amplified } & \multirow[t]{2}{*}{ c.35G > T,p.G12V } & $\begin{array}{l}\text { c. } 1796 \mathrm{C}> \\
\text { T,p.T599| }\end{array}$ \\
\hline & & & & $\begin{array}{l}\text { c. } 1805 \mathrm{C}> \\
\text { T.p.S602F }\end{array}$ \\
\hline 16 & Wild type & Non-amplified & Wild type & $\begin{array}{l}\text { c. } 1798 \mathrm{G}> \\
\text { A,p.V600M }\end{array}$ \\
\hline
\end{tabular}




\begin{tabular}{|c|c|c|c|c|}
\hline $\begin{array}{l}\text { Case } \\
\text { number }\end{array}$ & $\begin{array}{l}\text { Her2 gene } \\
\text { mutation }\end{array}$ & $\begin{array}{l}\text { Her2 gene } \\
\text { amplified }\end{array}$ & KRAS gene mutation & $\begin{array}{l}\text { BRAF gene } \\
\text { mutation }\end{array}$ \\
\hline 17 & $\begin{array}{l}\text { c. } 2908 \mathrm{C}> \\
\text { T,p.R970W }\end{array}$ & amplified & Wild type & Wild type \\
\hline 18 & $\begin{array}{l}\text { c. } 2912 A>G, p . \\
\text { E } 971 G\end{array}$ & amplified & Wild type & $\begin{array}{l}\text { c. } 1796 \mathrm{C}> \\
\text { T,p.T599| }\end{array}$ \\
\hline 19 & Wild type & amplified & Wild type & Wild type \\
\hline 20 & Wild type & amplified & $\begin{array}{l}\text { c.32C > T, p.A11V } \\
\text { c.37_38delinsAA,p.A13V } \\
\text { c.40G > A, p.V14I }\end{array}$ & $\begin{array}{l}\text { c. } 1805 \mathrm{C}> \\
\text { T,p.S602F }\end{array}$ \\
\hline
\end{tabular}

Using a pairwise comparison method, we identified that the prevalence of $B R A F$ gene mutation is more common than that of HER2 gene mutation ( $80 \%$ vs. 35\%; $p=0.022$, McNemar test). As well, the prevalence of $B R A F$ gene mutation is more common than that $H E R 2$ gene amplification $(80 \% v s .35 \% ; \mathrm{p}=$ 0.022 , McNemar test). Even though the mutation rate of $B R A F$ gene is numerically higher than that of KRAS gene, they both were not significantly different ( $80 \%$ vs. $60 \% ; \mathrm{p}=0.289$, McNemar test). (Fig. 2)

We identified that the dual set with $B R A F$ and $K R A S$ mutations occurred in 10 cases (50\%), the dual set with $B R A F$ mutation and HER2 amplification occurred in 4 cases (20\%), as well as the dual set with BRAF and $H E R 2$ mutations occurred in in 5 cases (25\%). Additionally, the triple set with BRAF mutation, KRAS mutation and HER2 amplification occurred in 2 case (10\%), the triple set with BRAF mutation, KRAS mutation and $H E R 2$ mutation occurred in 1 case (5\%), as well as the triple set with $B R A F$ mutation, $H E R 2$ mutation and HER2 amplification occurred in 2 case (10\%). Nonetheless, the quadruple set with BRAF mutation, HER2 amplification, HER2 mutation and KRAS mutation occurred in none (0\%). (Table 1 )

Adopting the newly classification scheme for $B R A F$ mutations to $\mathrm{MOC}$, we discovered 2 cases of class I $B R A F$ mutants including V600E $(\mathrm{n}=1)$ and $\operatorname{V600M}(\mathrm{n}=1)$; as well as 12 cases of class II BRAF mutants including A598V $(n=1)$, T599I $(n=10)$ and dual T599I/S602F $(n=1)$. No class III BRAF mutants were found. However, we identified 3 cases with the novel unclassified BRAF mutants, including single S602F $(n=2)$ and the dual T599I/S602F $(n=1)$ that are repeatedly counted. (Fig. 3)

\section{Discussion}

The BRAF (v-raf murine sarcoma viral oncogene homolog B1) gene is located on the long arm of chromosome 7 (7q34) and encodes for an 18-exon cytoplasmic protein, a serine/threonine protein kinase (B-Raf) which can be recruited to the membrane upon stimulation of $H E R 2$ receptor. BRAF is a serine/threonine protein kinase, which is an important signal transducer of the HER2 triggered RAS-RAFmitogen-activated protein kinase kinase $(M E K)$ - extracellular signal regulated kinase $(E R K)$ signaling pathway (also known as $R A S / R A F / M E K / E R K$ pathway or MAPK cascade). Active BRAF then activates 
MEK $1 / 2$ to phosphorylate ERK $1 / 2$, which leads to the expression of several downstream transcription factors that regulate cell growth, differentiation, and survival. $[4,16]$

We have already known that the early stage MOC usually has an excellent prognosis, but late stage MOC carries a poor outcome. [1] The encouraging success of $B R A F$ inhibitors that can successfully treat some melanoma patients with $B R A F(\mathrm{~V} 600)$ mutations has prompted us to investigate the $B R A F$ status and its therapeutic implication in advanced MOC. However, few studies have characterized its $B R A F$ oncogene status and its response to anti-BRAF drugs has not yet been comprehensively explored in MOC. Even though melanoma and $\mathrm{MOC}$ are different tumors, we imagine that the functional consequence and anti$B R A F$ effect of the tumors harboring $B R A F$ mutations might be similar. $[17,18]$

After merging the previously reported raw data of HER2 amplifications, HER2 mutations and KRAS mutations with the new information of $B R A F$, we identified that their corresponding frequencies are $35 \%$ for HER2 amplifications, $35 \%$ for HER2 mutations, $60 \%$ for $K R A S$ mutations and $80 \%$ for BRAF mutations in all 20 MOC Taiwanese patients (Table 1). [9, 10] Our findings also indicated that there was no significant difference in the frequency between BRAF mutations and KRAS mutations when they were compared. However, the frequency of $B R A F$ mutations was significant higher than that of HER2 amplifications and HER2 mutations, respectively. (Fig. 2)

Focusing on the basis of $B R A F$ variant together with HER2 amplifications, HER2 mutations, KRAS mutations within the HER2 triggered MAPK signaling pathway, we divided them into the BRAF-based dual, triple and quadruple sets, respectively. After that, we identified that the BRAF-based dual and triple sets are not uncommon except for the quadruple set (Table 1). Our data indicated that the coexisting mutations of these driver genes indeed occur in MOC, which might cause synergistic effects in tumorigenesis.

In our study, one case showing HER2 amplification, but wild-type HER2, KRAS and BRAF genes indicated that HER2 alone may in fact confer heightened sensitivity to existing anti-HER2 therapies (i.e. trastuzumab, lapatinib) because of dependency on the most upstream receptor tyrosine kinase (RTK) signaling. (Table 1, case no. 19) Moreover, high frequency of HER2 gene amplification $(\mathrm{n}=7)$ combined with HER2 mutation ( $\mathrm{n}=3 ; 42.86 \%$ \& Table 1 , case no. $4,17,18)$, with KRAS mutation $(\mathrm{n}=2 ; 28.58 \%$ \& Table 1 , case no. 1,20$)$ or with $B R A F$ mutation $(n=5 ; 71.43 \%$ \& Table 1 , case no. $1,4,12,18,20)$ can predict unresponsiveness or refractoriness to single HER2 inhibition as a result of the constitutive activation of the MAPK pathway downstream of HER2 signaling. On the other hand, we identified one case with $H E R 2$ non-amplification, $H E R 2$ wild-type, KRAS wild-type, but alternative $B R A F$ mutation (V600M). (Table 1, case no. 16) It is suggested that the downstream BRAF(V600) mutations alone might be enough to trigger continuous activation of MAPK signaling cascade, leading to tumorigenesis. The existing V600 BRAF inhibitors (i.e. Vemurafenib, dabrafeniband and encorafenib) may have benefit to some Taiwanese patients with primary MOC; however, the clinical evidence that currently exists to substantiate these claims are insufficient. 
Previous reports from other countries have shown that MOC has a lower frequency of BRAF mutation (2$20 \%)$. $[1,2,6]$ Our data revealed that the $B R A F$ missense mutation rate is relatively up to $80 \%(n=16 / 20)$ using the FemtoPath BRAF Mutation Screen Kit. This kit is a PCR-based test using proprietary primers which can selectively amplify the somatic mutations in activating segment of the BRAF gene, and suppresses the amplification of wild-type $B R A F$ gene in human genomic DNA. [13, 14] Although timeconsuming, DNA sequencing techniques are still the current gold standard for mutational testing.

Despite geographical, racial and ethnic differences, the following 5 standpoints explain the reason why the BRAF mutation rate of this study is higher than that of others. (1) We used the H-E (hematoxylin and eosin stain) based microdissection technique to obtain a high percentage of representative tumor parts from formalin-fixed paraffin-embedded (FFPE) tissues, which restricted our analysis to only those tumor cells that express a specific marker or have a specific gene mutation. (2) According to the manufacture's manual and the previous report of the Stuntmer PCR technology, this highly specific and sensitive mutation enrich technology can detect less than $1 \%$ (as little as 20-100 ng) of BRAFV600 variants within exon 15. Additionally, the neighboring mutation sites of V600 (amino acid range 591-620) can also be amplified at the same time. Based on the identical principle of Stuntmer PCR, all of the BRAF mutations detected may share the similar high sensitivity. (3) We used other 7 normal ovarian tissues as negative controls, but none of them revealed BRAF mutations using the same kit. (4) Furthermore, the prior report also demonstrates that a stuntmer can inhibit wild type template replication, thereby allowing for selective amplification of mutants in a non-sequence specific manner. (5) Even after three rounds of PCRs, the original wild-type signal group remained unaltered, demonstrating that the stuntmer does not alter the original sequence of the sample [12-14]. The above-mentioned (1)-(5) evidences support that the possibility of false positive BRAF gene mutations detected using FemtoPath BRAF Mutation Screen Kit is extremely low.

According to the new classification system for BRAF mutations, different classes can predict their matching clinical response to contemporary targeted therapies on the market and have important implications for future anti-BRAF development. [7] In our patient cohort, we detected 4 kinds of known BRAF missense variants, 2 of which were class I (V600E, V600M), 2 were class II (A598V, T599I) and none was class III. Additionally, we identified one novel BRAF variant (S602F) that has never been reported in accordance with the COSMIC database. Even though the biochemical and signaling mechanism of the new BRAF variant (S602F) has not yet been comprehensively studied, its predicted functional effect appeared to be probably damaging in accordance with the Polyphen-2 database. As well, we suspect that the $\mathrm{S} 602 \mathrm{~F}$ might be categorized as class II BRAF variant, because it is located in the activating segment of $B R A F$ kinase domain.

In summary, BRAF mutation is not uncommon in primary MOC of Taiwanese. When taken together with previous published data, we found that the activating BRAF mutation, HER2 amplification, HER2 mutation and $K R A S$ mutation were not mutually exclusive, but simultaneously independent. However, they may even have a synergistic effect in tumorigenesis. Even though our results are confident and 
comprehensive, the case number cohort was small. Further exploratory studies should be performed to validate these finding.

\section{Conclusions}

Despite possible underestimation in some other studies, our solid data demonstrated that BRAF gene mutation rate was $80 \%$ of primary $\mathrm{MOC}$ in Taiwanese patients, using the FemtoPath BRAF Mutation Screen Kit. One novel BRAF mutation (S602F) has been identified in this study. Unlike melanoma and papillary thyroid carcinoma, the most common BRAF mutation of $\mathrm{MOC}$ is the non-V600 class II variant with T599I $(n=11 / 20 ; 55 \%)$ in Taiwanese. So far, there are no effective targeted treatments available for patients who have tumors or diseases harboring non-V600 BRAF mutations. $[5,19]$ Our results highlight the importance of developing new anti-BRAF therapeutic options for such patients harboring non-V600 $B R A F$ mutations. Alternatively, it indicated that the potential treatment strategy of recurrent or metastatic MOC might favor dual BRAF plus MEK inhibitor or single MEK inhibitor rather than the existing anti-BRAF V600 class I inhibitors. Further studies are warranted to investigate the clinical benefits of class-specific therapy in BRAF-altered metastatic or advanced stage MOC patients.

\section{Abbreviations}

BRAF

Proto-oncogene B-Raf and v-Raf murine sarcoma viral oncogene homolog B

Kras

V-Ki-ras2 Kirsten rat sarcoma viral oncogene homolog gene

Her2

Human epidermal growth factor receptor 2 gene

MAPK

Mitogen-Activated Protein Kinases

\section{Declarations}

\section{Ethics approval and consent to participate:}

Our research was conducted in accordance with the International Conference on Harmonization (ICH) guidelines and compliant with all applicable regulations and the protection of human subjects for research, including review and approval by the Institutional Review Board of the Chung-Shan Medical University Hospital, Taichung, Taiwan.

\section{Consent for publication:}

Not applicable. 


\section{Availability of data and material:}

Please contact author for data requests.

\section{Competing interests:}

The authors declare that there are no competing interests.

\section{Funding}

Grant: CSH-2020-C-029.

\section{Authors' contributions:}

$\mathrm{CPH}$ and GTH conceived and designed the research; $\mathrm{YJL}$, WRC performed the experiments and wrote the manuscript. All authors read and approved the final manuscript.

\section{Acknowledgements:}

We are extremely grateful to Bernard A (HHS/OPHS) Schwetz, DVM, PhD for his expertise and patience in English correction and helpful comments.

\section{References}

1. Babaier A, Ghatage P. Mucinous Cancer of the Ovary: Overview and Current Status. Diagnostics. 2020;10:52.

2. Morice P, Gouy S, Leary A. Mucinous Ovarian Carcinoma. N Engl J Med. 2019;380(13):1256-66. doi:10.1056/NEJMra1813254.

3. Chao WR, Lee MY, Lin WL, Koo CL, Sheu GT, Han CP. Assessing the HER2 status in mucinous epithelial ovarian cancer on the basis of the 2013 ASCO/CAP guideline update. Am J Surg Pathol. 2014;38(9):1227-34. doi:10.1097/PAS.0000000000000268.

4. Matallanas $D$, Birtwistle $M$, Romano $D$, et al. Raf family kinases: old dogs have learned new tricks. Genes Cancer. 2011;2(3):232-60. doi:10.1177/1947601911407323.

5. Croce L, Coperchini F, Magri F, Chiovato L, Rotondi M. The multifaceted anti-cancer effects of BRAFinhibitors. Oncotarget. 2019;10(61):6623-40. doi:10.18632/oncotarget.27304. Published 2019 Nov 12.

6. Wong KK, Tsai CC, Gershenson DM. BRAF mutational analysis in ovarian tumors: recent perspectives. Pathology Laboratory Medicine International. 2015;7:75-82. https://doi.org/10.2147/PLMI.S64383. 
7. Dankner M, Rose AAN, Rajkumar S, Siegel PM, Watson IR. Classifying BRAF alterations in cancer: new rational therapeutic strategies for actionable mutations. Oncogene. 2018;37(24):3183-99. doi:10.1038/s41388-018-0171-x.

8. Lin WL, Kuo WH, Chen FL, Lee MY, Ruan A, Tyan YS, et al. Identification of the coexisting HER2 gene amplification and novel mutations in the HER2 protein overexpressed mucinous epithelial ovarian cancer. Ann Surg Oncol. 2011;18(8):2388e94.

9. Chang KL, Lee MY, Chao WR, Han CP. The status of Her2 amplification and Kras mutations in mucinous ovarian carcinoma. Hum Genomics. 2016;10(1):40. doi:10.1186/s40246-016-0096-9. Published 2016 Dec 28.

10. Chiu HH, Chao WR, Chen CK, Lee YJ, Lee MY, Han CP. The Her2 gene aberrations in mucinous ovarian carcinoma: Analysis of twenty-one cases. Taiwan J Obstet Gynecol. 2020;59(2):346-7. doi:10.1016/j.tjog.2020.01.031.

11. Chen CK, Huang JK. Universal insertion/deletion-enrich PCR. Taiwan J Obstet Gynecol. 2011;50(4):499-502. doi:10.1016/j.tjog.2011.10.017.

12. Huang J, Fan $L$, Wang $T$, et al. A new primer construction technique that effectively increases amplification of rare mutant templates in samples. BMC Biotechnol. 2019;19:62. https://doi.org/10.1186/s12896-019-0555-1.

13. FemtoPath BRAF. Mutation Screen Kit, http://femtopath.com/product/braf-mutation-screen-kit/, access on Aug 22, 2020.

14. Medaysis Ultra-Sensitive BRAF Mutation Detection Kit User Manual. https://nebula.wsimg.com/01261a59a59497505f303e48c5caba62? AccessKeyld=D7617A0F62844F16AE6E\&disposition=0\&alloworigin=1, access on Aug 22, 2020.

15. Adzhubei I, Jordan DM, Sunyaev SR. Predicting functional effect of human missense mutations using PolyPhen-2. Curr Protoc Hum Genet. 2013;Chap. 7:Unit7.20. doi:10.1002/0471142905.hg0720s76.

16. Giunta EF, De Falco V, Napolitano S, et al. Optimal treatment strategy for metastatic melanoma patients harboring BRAF-V600 mutations. Ther Adv Med Oncol. 2020;12:1758835920925219. doi:10.1177/1758835920925219. Published 2020 Jun 19.

17. Johnson DB, Dahlman KB. Class Matters: Sensitivity of BRAF-Mutant Melanoma to MAPK Inhibition. Clin Cancer Res. 2018;24(24):6107-9. doi:10.1158/1078-0432.CCR-18-1795.

18. Targeted Therapy Drugs for Melanoma Skin Cancer. American Cancer Society, cancer.org I 1.800.227.2345. https://www.cancer.org/content/dam/CRC/PDF/Public/8826.00.pdf.

19. Turski ML, Vidwans SJ, Janku F, et al. Genomically Driven Tumors and Actionability across Histologies: BRAF-Mutant Cancers as a Paradigm. Mol Cancer Ther. 2016;15(4):533-47. doi:10.1158/1535-7163.MCT-15-0643.

\section{Figures}


(a)

BRAF
ATG
1

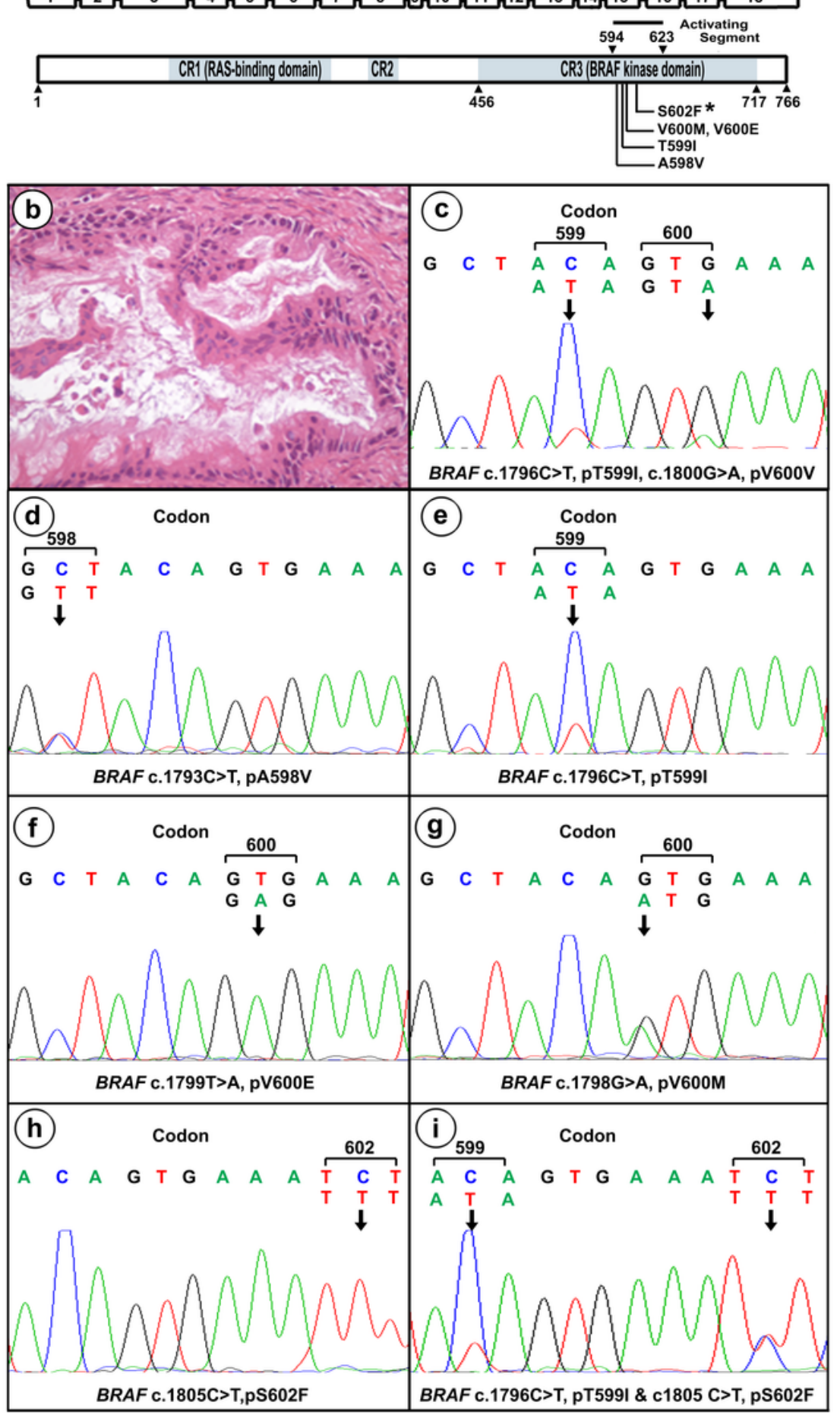

Figure 1

(a) BRAF is located on chromosome 7q34 and contains 18 exons with intervening sequences. The start codon (ATG) and stop codon (TGA) are indicated. Schematic drawings show that the detected BRAF missense mutations are located within the activation segment (exon 15) of the CR3 (conserved regions $3)$, kinase domain in MOC. An asterisk (*) indicates the novel finding in this report, not documented in the COSMIC database. (b) Representative case showing histopathologic picture with Hematoxylin-eosin 
stain, 400x. (c), (d), (e), (f), (g), (h), (i) DNA electropherograms depicting the various BRAF misense mutations by arrows, and validating by direct Sanger sequencing.

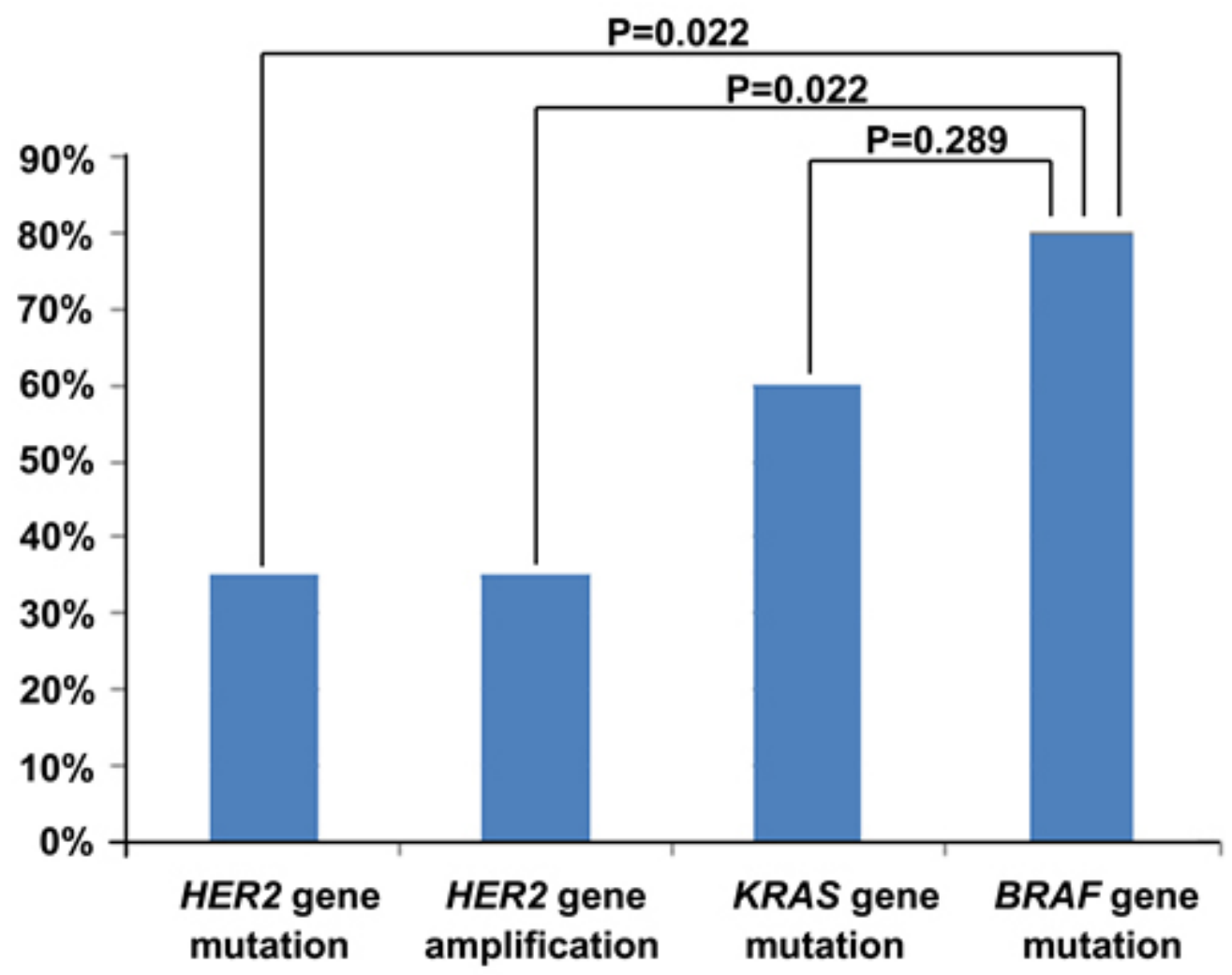

Figure 2

Pairwise comparison of frequencies of BRAF gene mutation vs. KRAS gene mutation; BRAF gene mutation vs. HER2 gene mutation; and BRAF gene mutation vs. HER2 gene amplification using McNemar test $(a=0.05)$. 


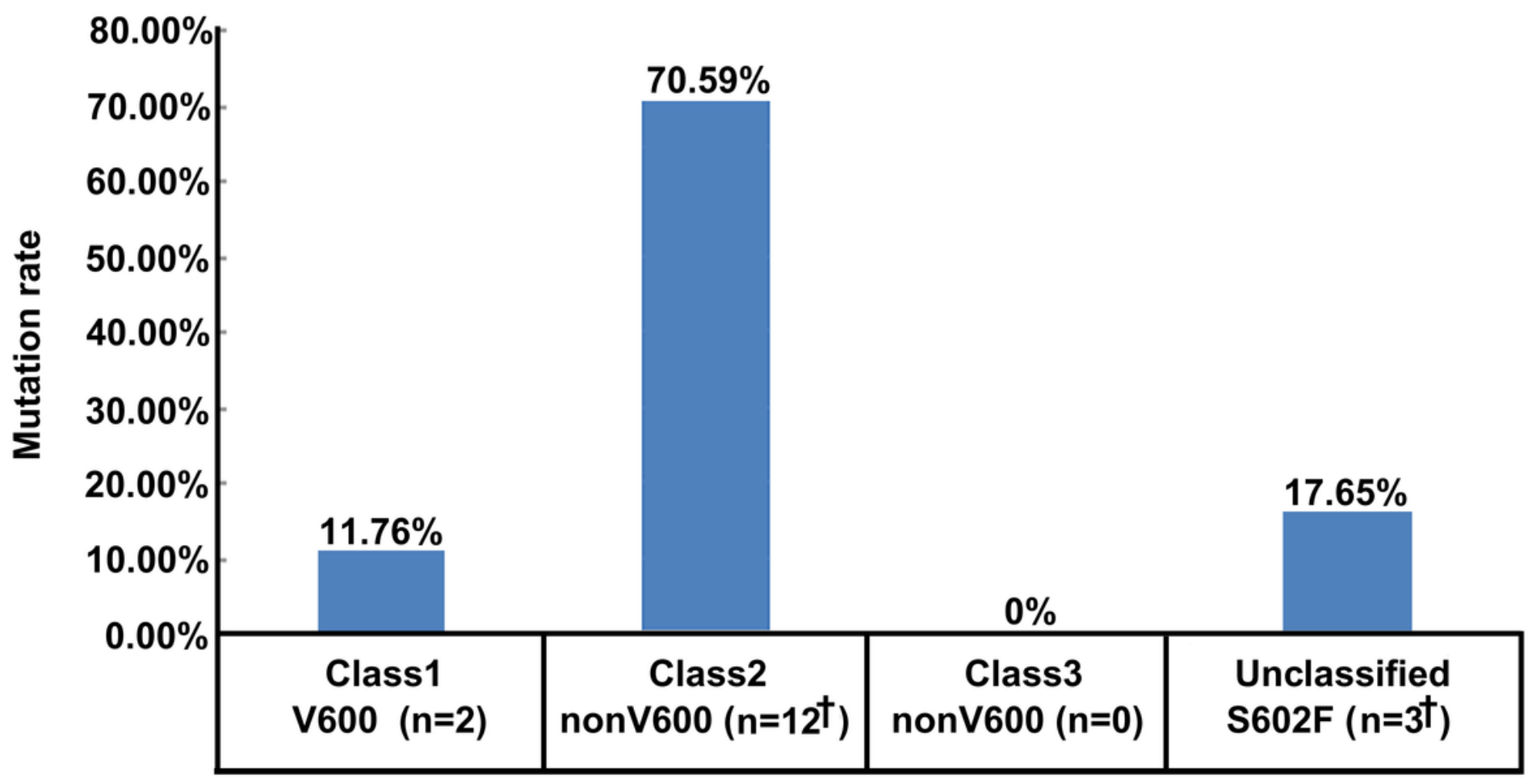

$B R A F$ mutation classification ( $\mathrm{n}$ : number of cases)

Figure 3

legend BRAF mutations are categorized into class-1 (high kinase activity, V600), class-2 (high or intermediate kinase activity, nonV600), class-3 (impaired BRAF kinase activity) and unclassified (unknown BRAF kinase activity). The " $n$ " means number of cases existed. The symbol " $\dagger$ "means one case with dual missense mutations (T599I and S602F) of BRAF that are counted twice in class 2 and unclassified categories, disparately. 